\title{
Short-term outcomes in robot-assisted compared to laparoscopic colon cancer resections: a systematic review and meta-analysis
}

\author{
Pedja Cuk ${ }^{1,3}$ - Mie Dilling Kjær ${ }^{2} \cdot$ Christian Backer Mogensen ${ }^{3} \cdot$ Michael Festersen Nielsen $^{1,3}$. \\ Andreas Kristian Pedersen ${ }^{3} \cdot$ Mark Bremholm Ellebæk $^{2}$
}

Received: 12 July 2021 / Accepted: 17 October 2021 / Published online: 1 November 2021

(c) The Author(s) 2021

\begin{abstract}
Background Robot-assisted surgery is increasingly adopted in colorectal surgery. However, evidence for the implementation of robot-assisted surgery for colon cancer is sparse. This study aims to evaluate the short-term outcomes of robot-assisted colon surgery (RCS) for cancer compared to laparoscopic colon surgery (LCS).

Methods Embase, MEDLINE, and Cochrane Library were searched between January 1, 2005 and October 2, 2020. Randomized clinical trials and observational studies were included. Non-original literature was excluded. Primary endpoints were anastomotic leakage rate, conversion to open surgery, operative time, and length of hospital stay. Secondary endpoints were surgical efficacy and postoperative morbidity. We evaluated risk of bias using RoB2 and ROBINS-I quality assessment tools. We performed a pooled analysis of primary and secondary endpoints. Heterogeneity was assessed by $I^{2}$, and possible causes were explored by sensitivity- and meta-regression analyses. Publication bias was evaluated by Funnel plots and Eggers linear regression test. The level of evidence was assessed by GRADE.

Results Twenty studies enrolling 13,799 patients (RCS 1740 (12.6\%) and LCS 12,059 (87.4\%) were included in the metaanalysis that demonstrated RCS was superior regarding: anastomotic leakage (odds ratio $(\mathrm{OR})=0.54,95 \% \mathrm{CI}[0.32,0.94]$ ), conversion $(\mathrm{OR}=0.31,95 \% \mathrm{CI}[0.23,0.41])$, overall complication rate $(\mathrm{OR}=0.85,95 \% \mathrm{CI}[0.73,1.00])$ and time to regular $\operatorname{diet}(\mathrm{MD}=-0.29,95 \% \mathrm{CI}[-0.56,0.02])$. LCS proved to have a shortened operative time compared to RCS $(\mathrm{MD}=42.99$, $95 \%$ CI $[28.37,57.60])$. Level of evidence was very low according to GRADE.

Conclusion RCS showed advantages in colonic cancer surgery regarding surgical efficacy and morbidity compared to LCS despite a predominant inclusion of non-RCT with serious risk of bias assessment and a very low level of evidence.
\end{abstract}

Keywords Robot-assisted surgery $\cdot$ Colon cancer $\cdot$ Laparoscopy $\cdot$ Minimally invasive surgery

Minimally invasive technology has undergone a rapid advancement within the last 20 years introducing robotassisted colon surgery (RCS). First reports of RCS were described by Weber et al. [1]. The method was implemented in order to simplify dissection in the narrow pelvis and improve ergonomics for the surgeon. Due to a technological progress within the last decade, robot-assisted surgery

Pedja Cuk

pedja.cuk@rsyd.dk

1 Surgical Department, University Hospital of Southern Jutland, Kresten Philipsens Vej 15, 6200 Aabenraa, Denmark

2 Research Unit for Surgery, Odense University Hospital and University of Southern Denmark, Odense, Denmark

3 Institute of Regional Health Research, University of Southern Denmark, Odense, Denmark has minimized tremor and enhanced camera guidance, which may have improved and simplified the dissection [2-5]. Laparoscopic colon surgery (LCS) is associated with a reduced overall morbidity, shortened hospital stay, minimized analgesics consumption, faster recovery of bowel function and oral intake compared to open surgery [6-8]. The method results in less manipulation of the intra-abdominal organs and contributes to a lower surgical stress response [9-11]. To our knowledge benefits of RCS compared to LCS have not been examined in randomized controlled trials with the exception of Park et al. [12]. No difference in intra- or postoperative outcomes was demonstrated when the RCS was compared to LCS in a study based on a small number of patients $(n=70)$ [12]. However, a prolonged operation time and higher procedure costs were observed in RCS. 
Present literature that compares RCS and LCS includes a mixed population of benign and malignant colorectal surgery [13-15]. Systematic reviews and meta-analyses have shown a favorable outcome for the intracorporeal anastomosis technique when assessed for intra- versus extracorporeal anastomosis technique in colectomies for right-sided colon cancer [16]. Besides lower morbidity rates in rightsided colectomies performed by RCS versus LCS in a heterogenous population, to our knowledge, no meta-analyses has been conducted to determine overall effects of RCS in colonic cancer surgery $[15,16]$. It would be relevant to perform a systematic review and, if possible, a meta-analysis on the effectiveness and safety of RCS versus LCS in a patient cohort with only colon cancer. In addition to the main analysis, a supplemental sensitivity- and meta-regression analysis is likely to be conducted to demonstrate any potential subgroup-influence on the overall analysis.

We aim to evaluate the short-term outcomes of robotassisted colon resection for cancer compared to conventional laparoscopy in this systematic review and meta-analysis. Primary endpoints are anastomotic leakage rate, conversion to open surgery, operative time and length of hospital stay.

\section{Methods}

The study was performed in accordance with the Preferred Reporting Items for Systematic Reviews and Meta-Analyses (PRISMA) guidelines and Meta-analysis of Observational Studies in Epidemiology (MOOSE) checklist [17, 18] (Supplemental Digital Content Material 1, Tables 1,2). The first author registered the study protocol in PROSPERO, University of York (CRD42020211681).

\section{Search strategy}

A systematic literature search was performed with assistance of a medical research librarian, University of Southern Denmark in databases: Embase, MEDLINE, and Cochrane Library (CENTRAL). Following terms were included in the search strategy: "colonic neoplasms", "colon cancer", "colon tumor", "colon carcinoma", "laparoscopy" and "robot-assisted surgery". The exact literature search strategy included articles published between January 1, 2005 and October 2, 2020, provided in Supplemental Digital Content Material 1, Fig. 1. The time frame was selected as the first case series of RCS were published in 2002 [1], and we expected high-volume comparative studies on LCS and RCS to be relatively sparse within the first four years due to gradual implementation. Two independent authors (PC and MD) screened and selected all articles. In case of disagreement, a third independent senior author (MBE) was available for consultation. The first author imported the literature from databases into the review program Covidence ${ }^{\circledR}$, Covidence systematic review software, Veritas Health Innovation, Melbourne, Australia. A manual search for additional relevant articles was obtained from the reference list of included studies. Literature not available in the searched databases and by article review was obtained through direct correspondence to the main authors.

\section{Eligibility criteria}

Inclusion criteria were (1) comparative literature for elective colon cancer resections performed robot-assisted and laparoscopically, (2) colorectal studies if results were reported independently for colon surgery, and (3) further, we arbitrarily decided that studies must include a minimum 15 patients in both groups. Only studies published in English were included. Included study designs were randomized clinical trials (RCT) and observational studies. A preliminary search had indicated only few RCTs existed of that time, which to a modest degree would increase the level of evidence in a comprehensive systematic review and metaanalysis. The following studies were excluded in case of: duplicates, abstracts, cover letters, editorials, reviews, commentaries, case reports and pediatric studies. Studies based on a cohort of palliative colorectal resections, combined benign and malignant colorectal surgery not reporting the results independently or in case of emergent surgery were also excluded.

\section{Outcomes and data extraction}

Primary outcomes were anastomotic leakage rate, conversion from robot-assisted or laparoscopic to open surgery, operative time and length of hospital stay. Secondary outcomes included overall complication rates, time to first flatus and oral diet, intraoperative blood loss, rate of postoperative bleeding, surgical site infection, intra-abdominal abscess formation, postoperative bowel obstruction, the total amount of harvested lymph nodes, 30 days mortality, Clavien-Dindo classification I-V, surgical and medical complications [19]. Data were extracted by PC into a priori developed data extraction form. Following extraction were performed regarding study data: authors, year of publication, study design, country of study, study period, risk of bias and quality of evidence assessments. We extracted following demographic data: sex, age, BMI, ASA-score $\geq 3$, T-stage $\geq 3$, location of tumor, type of surgical procedure and if previous abdominal surgery was performed. Data referring to predefined primary and secondary endpoints were also extracted. 


\section{Risk of bias assessment and quality of evidence (GRADE)}

Three reviewers (PC, MD and AKP) performed the risk of bias assessment by using the Cochrane handbook risk of bias (RoB2-2019) and (ROBINS-I tool-2016) [20, 21]. The first author used an electronic program, Robvis, to create quality assessment and weighted bar plots to provide better visualization of the individual studies [22]. PC and AKP assessed the certainty of evidence by applying "The Grading of Recommendations Assessment, Development and Evaluation (GRADE)" approach [23]. A summary of findings table was performed on the most important clinical outcomes with the assistance of GRADEpro GDT software (GRADEpro GDT: GRADEpro Guideline Development Tool [software]. McMaster University, 2020 (developed by Evidence Prime, Inc.). Available from https://gradepro.org).

\section{Statistical analysis}

For binary variables, odds ratios (OR) and corresponding 95\% confidence interval were calculated for each study. Continuous variables reported in median and range, were converted to mean and standard deviation as suggested by Hozo et al. [24]. Subsequently, mean differences and their corresponding $95 \%$ confidence intervals were calculated for each study. We used Higgins' I square statistics $\left(I^{2}\right)$ in order to examine the variation in heterogeneity across studies [25]. Values $<30 \%$ were associated with low heterogeneity, $>50 \%$ with substantial heterogeneity, while studies $>75 \% I^{2}$ were considerable heterogenic, as described in the Cochrane handbook for systematic reviews of interventions [26]. We used a fixed-effect model (Mantel-Haenszel method) in studies associated with low heterogeneity $(<50 \%)$. Random-effect models were utilized according to Der Simonian-Laird method in studies with high heterogeneity $(>50 \%)$. A $p$-value $<0.05$ was considered as statistically significant. We performed subgroup analyses and computed results from right-sided colectomies separately. In order to explain a possible implication of substantial and considerable heterogeneity ( $>50 \%$ ) found in our primary and secondary outcomes from the main analysis, we conducted a sensitivity analysis on right-sided resections. By visual inspection of forest and funnel plots, we identified and excluded outlier studies with extreme effect sizes. A random-effects meta-regression analysis was performed to examine a possible moderator effect on primary outcomes. We used baseline study and clinical moderators (year of study publication, age, male sex, BMI, T-stage $\geq 3$, anastomosis techniques (intra- or extracorporeal), and history of previous abdominal surgery). Publication bias were assessed by visual examination for any possible asymmetry presented in funnel plots and Egger's linear regression test [27, 28]. A significance level of $(p<0.05)$ was correlated to asymmetry and signs of publication bias. All statistical analysis were performed with STATA—StataCorp. 2019. Stata Statistical Software: Release 16. College Station, TX: StataCorp LLC in cooperation with a biostatistician (AKP).

\section{Results}

\section{Systematic review}

The systematic literature search yielded 1321 articles. After removal of duplicates, 966 articles were screened for title and abstract. Of these, 146 articles were included for full text screening, and subsequently 20 articles were included in the quantitative analysis $[12,29-31,33,35,36,38,39$, 41-51]. The complete search strategy is presented according to PRISMA guidelines in Fig. 1.

\section{Study characteristics}

A total of 1862 patients (13\%) underwent RCS and 12,231 (87\%) LCS. The majority of the included studies were of observational design [29-51] in addition to one RCT study by Park et al. [12]. Table 1 shows an overview of the included studies.

\section{Risk of bias assessment}

The RCT study by Park had a low risk of bias regarding randomization, missing outcome data and the selection of reported results. There was a moderate risk of bias related to deviations from intended interventions and measurements of the outcome. Due to the heterogeneous cohort of included patients from the observational studies, there was a predominantly serious risk of bias regarding confounding and selection of participants, as presented in Supplemental Digital Content Material 2, Fig. 1.

\section{Meta-analysis}

\section{Patient demographic}

We included 20 out of 24 studies from the systematic literature search in the meta-analysis [12, 29-31, 33, 35, 36, 38, $39,41-51]$ with a total of 13,799 patients [1740 (12.6\%) underwent RCS and 12,059 (87.4\%) LCS]. Significant differences in preoperative patient characteristics were determined between the RCS and LCS group regarding previous abdominal surgery [12, 29-31, 33, 35, 38, 41, 43, 44, 48, 49, 51] and male gender [12, 29-31, 33, 35, 36,38, 39, 41-46, 48-51]. Table 2 summarizes patient demographics. There 


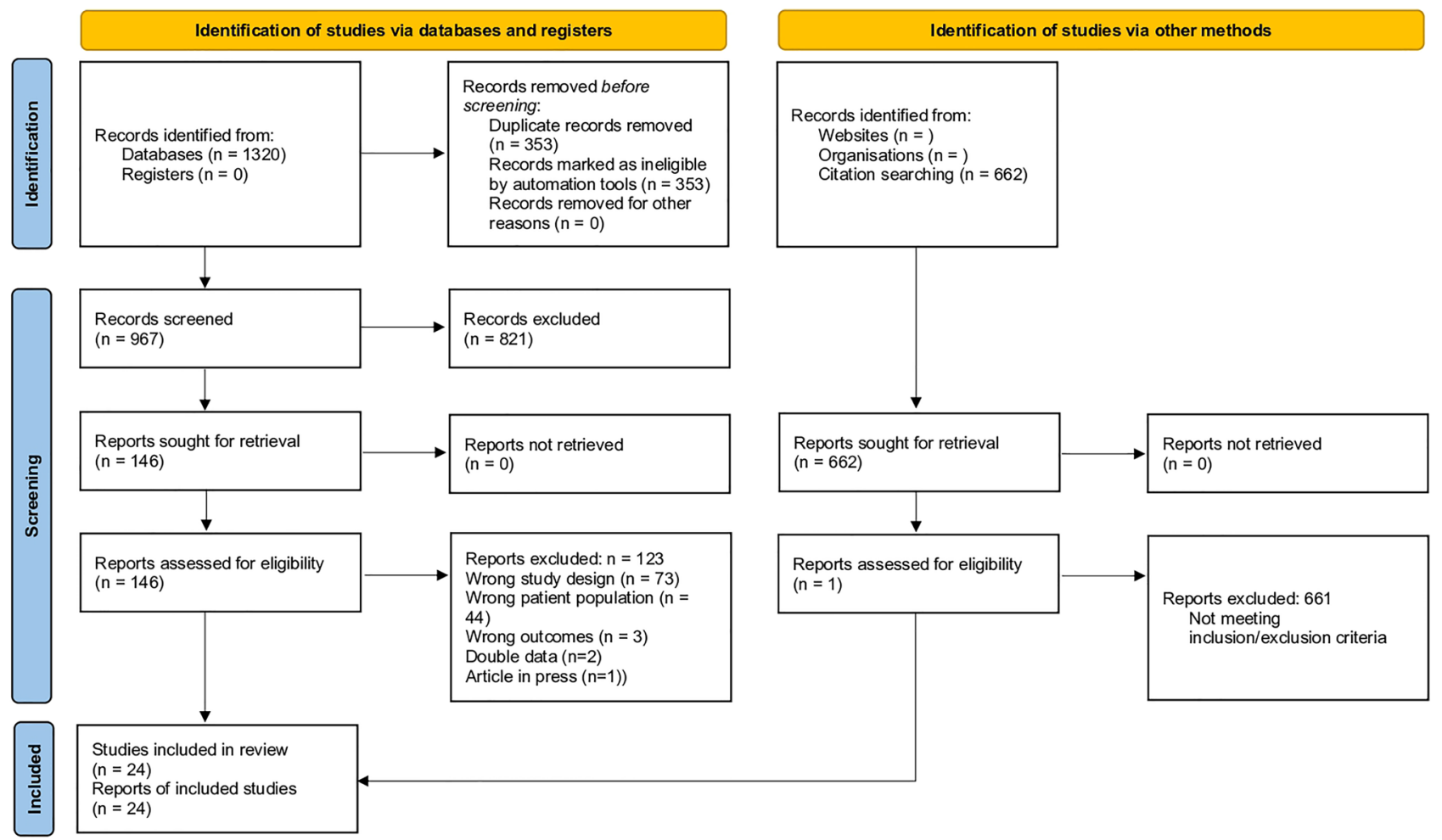

Fig. 1 PRISMA flowchart of study selection process

was no statistically significant difference in preoperative characteristics between the two groups regarding age, BMI, tumor stage (T3 $\geq 3$ ) or ASA-score $\geq 3$.

\section{Primary outcome: anastomotic leakage}

Anastomotic leakage rate was reported in 15 studies [12, 29-31, 33, 36, 38, 41-44, 47-49, 51], with 16 events out of $970(1.65 \%)$ in the RCS group and 41 out of 1310 (3.13\%) in LCS group with a significant difference in favor of RCS $\left(\mathrm{OR}=0.54,95 \% \mathrm{CI}[0.32,0.94], I^{2}=0 \%, p=0.03\right)$. There was a tendency for a reduction in anastomotic leakage rate in the RCS group especially after year 2018 [41, 44, 47-49] according to Fig. 2.

\section{Primary outcome: conversion to open surgery}

Conversion rates were reported in 17 studies [12, 29-31, 33, $35,38,39,41-45,47-49,51]$. There was an advantage for lower conversion rates in RCS versus LCS group $(\mathrm{OR}=0.31$, $95 \%$ CI $\left.[0.23,0.41], I^{2}=41.10 \%, \mathrm{p}=0.00\right)$, see Fig. 3 .

\section{Primary outcome: operative time}

Operative time was prolonged in RCS compared to LCS group $\left(\mathrm{MD}=42.99,95 \%\right.$ CI $[28.37,57.60], I^{2}=96.99 \%$, $p=0.00$ ). During the inclusion period the operative time for RCS was unchanged and showed no decreasing tendency among included studies $(n=19)$ [12, 29-31, 33, 35, 36, 38, 39, 41-44, 46-51]. Forest plot of operative time is presented in Supplemental Digital Content Material 3, Fig. 1.

\section{Primary outcome: length of hospital stay}

Length of hospital stay was reported in 17 studies [12, 29-31, 33, 35, 36, 38, 41-44, 46, 48-51] presented in Fig. 4, and did not differ significantly between the two surgical methods (MD $=-0.58,95 \%$ CI $[-1.37,0.21], I^{2}=91.10 \%$, $p=0.15)$.

\section{Secondary outcomes}

Data from overall complication rates were reported in 20 studies $[12,29-31,33,35,36,38,39,41-51]$ in which 303 complications out of $1740(17.41 \%)$ occurred in the RCS group and 2051 out of $12,059(17.01 \%)$ in LCS group. There was a significant lower difference in the pooled OR regarding the overall complication rate in favor of RCS $(\mathrm{OR}=0.85,95 \%$ CI $\left.[0.73,1.00], I^{2}=9.75 \%, p=0.04\right)$. A total of 11 studies $[12,29,30,33,35,38,41-43,49,50]$ reported a statistically significant difference in time to regular diet in favor of RCS $\left(\mathrm{MD}=-0.29,95 \%\right.$ CI $[-0.56,-0.02], I^{2}=58.17 \%$, 
Table 1 Study characteristics

\begin{tabular}{|c|c|c|c|c|c|c|}
\hline $\begin{array}{l}\text { Author country } \\
\text { Y.O. publ. study } \\
\text { period }\end{array}$ & $\begin{array}{l}\text { N.O. patients } \\
\text { RCS/LCS }\end{array}$ & $\begin{array}{l}\text { Anastomosis } \\
\text { technique, RCS/ } \\
\text { LCS (EC/IC/ } \\
\text { NA), } n(\%)\end{array}$ & $\begin{array}{l}\text { Age (years), mean } \pm \text { SD, } \\
\text { RCS/LCS }\end{array}$ & $\begin{array}{l}\text { BMI, mean }\left(\mathrm{kg} / \mathrm{m}^{2}\right) \mathrm{RCS} / \\
\text { LCS }\end{array}$ & Study type & Tumor location \\
\hline $\begin{array}{l}\text { Bertani, Italy, } \\
\text { 2011, 2009- } \\
2010[29]\end{array}$ & $34 / 30$ & IC and EC & $62.5 \pm 8.4 / 62.0 \pm 10.2$ & $26.1 \pm 3.71 / 24.6 \pm 3.54$ & Prospective & $\begin{array}{l}\text { Right and left } \\
\text { colon }\end{array}$ \\
\hline $\begin{array}{l}\text { Park, Korea, } \\
\text { 2012, 2009- } \\
2011 \text { [12] }\end{array}$ & $35 / 35$ & $\begin{array}{l}\text { RCS - EC: } \\
5 \text { (14), } \\
\text { RCS - IC: } \\
30(86), \\
\text { LCS - EC: } \\
28(80), \\
\text { LCS - IC: } 7 \\
(20)\end{array}$ & $62.8 \pm 10.5 / 66.5 \pm 11.4$ & $24.4 \pm 2.5 / 23.8 \pm 2.7$ & RCT & Right colon \\
\hline $\begin{array}{l}\text { Lim, Korea, } \\
\text { 2012, 2006- } \\
2008[30]\end{array}$ & $34 / 146$ & Only EC & $59.6 \pm 8.4 / 59.7 \pm 11.5$ & $24.8 \pm 2.1 / 23.8 \pm 3.8$ & Retrospective & Left colon \\
\hline $\begin{array}{l}\text { Helvind, Den- } \\
\text { mark, 2013, } \\
\text { 2009-2012 } \\
\text { [31] }\end{array}$ & $101 / 162$ & Only EC & $72.2(39-93) / 75.3(47-96)$ & $\begin{array}{l}24.6(16.8-36.0) / 24.9 \\
\quad(15.2-45.7)\end{array}$ & Retrospective & $\begin{array}{l}\text { Right and left } \\
\text { colon }\end{array}$ \\
\hline $\begin{array}{l}\text { Morpurgo, } \\
\text { Italy, 2013, } \\
2008-2012 \\
{[32]}\end{array}$ & $48 / 48$ & $\begin{array}{l}\text { RCS - IC: } \\
\quad 48 \text { (50), } \\
\text { LCS - EC: } 48 \\
(50)\end{array}$ & $68.8 \pm 8 / 74 \pm 11$ & $25 \pm 3.5 / 28 \pm 4$ & Retrospective & Right colon \\
\hline $\begin{array}{l}\text { de'Angelis, } \\
\text { France, 2015, } \\
\text { 2010-2014 } \\
\text { [33] }\end{array}$ & $22 / 22$ & Only EC & $72.18 \pm 10.79 / 71 \pm 10.14$ & $24.12 \pm 2.64 / 24.28 \pm 2.7$ & Prospective & Transverse colon \\
\hline $\begin{array}{l}\text { Guerrieri, } \\
\text { Italy, 2015, } \\
\text { 2013-2014 } \\
\text { [34] }\end{array}$ & $24 / 23$ & $\begin{array}{l}\text { RCS - EC: } \\
5(21), \\
\text { RCS - IC: } \\
19(79), \\
\text { LCS - EC: } \\
11(48), \\
\text { LCS - IC: } 12 \\
(52)\end{array}$ & $69.5(57-80) / 65.5(57-75)$ & $26(23-28) / 26.5(23-29)$ & Retrospective & $\begin{array}{l}\text { Right and left } \\
\text { colon }\end{array}$ \\
\hline $\begin{array}{l}\text { Kang, Korea, } \\
\text { 2016, 2007- } \\
2011[35]\end{array}$ & $20 / 43$ & Only EC & $66.0 \pm 9.6 / 65.7 \pm 13.2$ & $23.5 \pm 2.4 / 23.0 \pm 3.0$ & Retrospective & Right colon \\
\hline $\begin{array}{c}\text { Jung, Korea, } \\
2016,2007- \\
2013[36]\end{array}$ & $51 / 161$ & NR & $60.2 \pm 9.5 / 60.3 \pm 11.3$ & $23.5 \pm 3.0 / 23.2 \pm 2.8$ & Retrospective & $\begin{array}{l}\text { Right and left } \\
\text { colon }\end{array}$ \\
\hline $\begin{array}{l}\text { de'Angelis, } \\
\text { France, 2016, } \\
\text { 2012-2015 } \\
{[37]}\end{array}$ & $30 / 50$ & Only EC & $71 \pm 8.5 / 71.1 \pm 12.92$ & $26.43 \pm 3.21 / 25.26 \pm 4.19$ & Prospective & Right colon \\
\hline $\begin{array}{l}\text { Cardinali, } \\
\text { Italy, 2016, } \\
\text { 2013-2015 } \\
{[38]}\end{array}$ & $30 / 60$ & $\begin{array}{l}\text { RCS - IC: } \\
\quad 30 \text { (33), } \\
\text { LCS - EC: } 60 \\
(67)\end{array}$ & $68.67 \pm 12.93 / 70.78 \pm 9.56$ & $25.44 \pm 4.30 / 26.36 \pm 3.17$ & Retrospective & Right colon \\
\hline $\begin{array}{l}\text { Widmar, } \\
\text { USA, 2017, } \\
\text { 2012-2014 } \\
{[39]}\end{array}$ & $119 / 163$ & NR & $68(58-77) / 64(54-75)$ & $28(24-32) / 29(25-32)$ & Retrospective & Right colon \\
\hline $\begin{array}{l}\text { Kim, Korea, } \\
2018,2012- \\
2017[40]\end{array}$ & $20 / 51$ & Only EC & $58 \pm 10 / 56 \pm 13$ & $25.5 \pm 3.8 / 24 \pm 3.0$ & Retrospective & Left colon \\
\hline
\end{tabular}


Table 1 (continued)

\begin{tabular}{|c|c|c|c|c|c|c|}
\hline $\begin{array}{l}\text { Author country } \\
\text { Y.O. publ. study } \\
\text { period }\end{array}$ & $\begin{array}{l}\text { N.O. patients } \\
\text { RCS/LCS }\end{array}$ & $\begin{array}{l}\text { Anastomosis } \\
\text { technique, RCS/ } \\
\text { LCS (EC/IC/ } \\
\mathrm{NA}), n(\%)\end{array}$ & $\begin{array}{l}\text { Age (years), mean } \pm \text { SD, } \\
\text { RCS/LCS }\end{array}$ & $\begin{array}{l}\text { BMI, mean }\left(\mathrm{kg} / \mathrm{m}^{2}\right) \mathrm{RCS} / \\
\text { LCS }\end{array}$ & Study type & Tumor location \\
\hline $\begin{array}{l}\text { Scotton, Italy, } \\
2018,1998- \\
2017 \text { [41] }\end{array}$ & $206 / 160$ & $\begin{array}{l}\text { RCS - IC: } \\
206(56), \\
\text { LCS - EC: } \\
160(44)\end{array}$ & $70.1 \pm 11.7 / 70.3 \pm 12.7$ & $26.0 \pm 3.7 / 25.6 \pm 5.9$ & Retrospective & Right colon \\
\hline $\begin{array}{l}\text { Ngu, Singa- } \\
\text { pore, 2018, } \\
2015-2017 \\
{[42]}\end{array}$ & $16 / 16$ & Only IC & $68.6 \pm 10.9 / 69.6 \pm 9.6$ & $23.7 \pm 3.8 / 24.7 \pm 4.2$ & Retrospective & Right colon \\
\hline $\begin{array}{l}\text { Spinoglio, } \\
\text { Italy, 2018, } \\
2005-2013 \\
{[43]}\end{array}$ & $100 / 100$ & Only IC & $71.2 \pm 10.2 / 71.2 \pm 10.6$ & $25.1 \pm 4 / 25.8 \pm 4.4$ & Retrospective & Right colon \\
\hline $\begin{array}{l}\text { Mégevand, } \\
\text { Italy, 2018, } \\
2010-2015 \\
{[44]}\end{array}$ & $50 / 50$ & $\begin{array}{l}\text { RCS - IC: } \\
49 \text { (98), } \\
\text { RCS - EC: } 1 \\
\text { (2), LCS - IC: } \\
\text { 18 (36), } \\
\text { LCS - EC: } 32 \\
(64)\end{array}$ & $70.3 / 69.6$ & $26.2 / 25.25$ & Retrospective & Right colon \\
\hline $\begin{array}{l}\text { Fransgaard, } \\
\text { Denmark, } \\
2018,2010- \\
2015 \text { [45] }\end{array}$ & $511 / 8104$ & NR & $\mathrm{NE}$ & $\mathrm{NE}$ & Retrospective & $\begin{array}{l}\text { Right and left } \\
\text { colon/rectum }\end{array}$ \\
\hline $\begin{array}{l}\text { Haskins, } \\
\text { USA, 2018, } \\
\text { 2012-2014 } \\
\text { [46] }\end{array}$ & $89 / 2405$ & NR & $68.9 \pm 11.8 / 68.3 \pm 12.2$ & $29.3 \pm 6.3 / 28.5 \pm 6.3$ & Retrospective & Right colon \\
\hline $\begin{array}{l}\text { Polat, Nether- } \\
\text { lands, 2019, } \\
\text { 2014-2017 } \\
\text { [47] }\end{array}$ & $129 / 138$ & $\begin{array}{l}\text { RCS - IC: } \\
129(48), \\
\text { LCS - IC + EC: } \\
138(52) \\
\text { depending on } \\
\text { the surgeon's } \\
\text { choice }\end{array}$ & $\mathrm{NE}$ & $\mathrm{NE}$ & Retrospective & $\begin{array}{l}\text { Right and left } \\
\text { colon/rectum }\end{array}$ \\
\hline $\begin{array}{l}\text { Merola, Italy, } \\
\text { 2019, 2012- } \\
2017 \text { [51] }\end{array}$ & $94 / 94$ & $\begin{array}{l}\text { RCS - IC: } \\
94(50), \\
\text { LCS - IC: } 94 \\
(50)\end{array}$ & $69.41 \pm 10.31 / 72.09 \pm 9.54$ & $26.94 \pm 4.91 / 27.97 \pm 5.73$ & Retrospective & Right colon \\
\hline $\begin{array}{l}\text { Yozgatli, } \\
\text { Turkey, 2019, } \\
\text { 2015-2017 } \\
\text { [48] }\end{array}$ & $35 / 61$ & $\begin{array}{l}\text { RCS-IC: } 35 \\
\text { (36), LCS - IC } \\
\text { and EC } 61 \\
\text { (54) based } \\
\text { on surgeon's } \\
\text { choice }\end{array}$ & $65 \pm 13 / 65 \pm 13$ & $29 \pm 5 / 27 \pm 5$ & Retrospective & Right colon \\
\hline $\begin{array}{l}\text { Ozben, Turkey, } \\
\text { 2020, 2011- } \\
\text { 2018 [49] }\end{array}$ & $38 / 80$ & $\begin{array}{l}\text { RCS - IC: } \\
33 \text { (86.8), } \\
\text { RCS - EC: } \\
4(10.5), \\
\text { RCS - NA: } \\
1 \text { (2.6), } \\
\text { LCS - IC: } \\
16(20.0), \\
\text { LCS - EC: } \\
60(75), \\
\text { LCS - NA: } 4 \\
(5.0)\end{array}$ & $62.3 \pm 15.7 / 64.1 \pm 15.5$ & $25.3 \pm 4.5 / 26.7 \pm 5.7$ & Retrospective & Transverse colon \\
\hline
\end{tabular}


Table 1 (continued)

\begin{tabular}{|c|c|c|c|c|c|c|}
\hline $\begin{array}{l}\text { Author country } \\
\text { Y.O. publ. study } \\
\text { period }\end{array}$ & $\begin{array}{l}\text { N.O. patients } \\
\text { RCS/LCS }\end{array}$ & $\begin{array}{l}\text { Anastomosis } \\
\text { technique, RCS/ } \\
\text { LCS (EC/IC/ } \\
\text { NA), } n(\%)\end{array}$ & $\begin{array}{l}\text { Age (years), mean } \pm \text { SD, } \\
\text { RCS/LCS }\end{array}$ & $\begin{array}{l}\text { BMI, mean }\left(\mathrm{kg} / \mathrm{m}^{2}\right) \mathrm{RCS} / \\
\text { LCS }\end{array}$ & Study type & Tumor location \\
\hline $\begin{array}{l}\text { Ceccarelli, } \\
\text { Italy, 2020, } \\
2014-2019 \\
{[50]}\end{array}$ & $26 / 29$ & Only IC & $69.1 \pm 9.4 / 75.0 \pm 11.7$ & $24.4 \pm 3.8 / 24.2 \pm 2.8$ & Retrospective & Right colon \\
\hline $\begin{array}{c}\text { Total N.O. } \\
\text { patients }\end{array}$ & $\begin{array}{l}1862 \\
\quad(13 \%) / 12,231 \\
(87 \%)\end{array}$ & & & & & \\
\hline
\end{tabular}

Y.O. publ year of publication, N.O. number of, RCS robot-assisted colon surgery, LCS laparoscopic colon surgery, EC extracorporeal, IC intracorporeal, $N A$ no anastomosis, $R C T$ randomized controlled trial, $S D$ standard deviation, $N R$ not reported, $N E$ not estimable

Table 2 Meta-analysis of patient characteristics in the included studies

\begin{tabular}{lllllrl}
\hline Outcome & N.O. Studies & RCS & LCS & OR/MD 95\% CI & $I^{2}(\%)$ & $p$-value \\
\hline Age (SD) & 17 & $66.87(10.31)$ & $67.69(10.81)$ & $-0.67[-2.46,1.11]$ & 80.85 & 0.46 \\
BMI (SD) & 17 & $25.54(3.58)$ & $25.49(4.03)$ & $0.02[-0.47,0.51]$ & 63.54 & 0.95 \\
Male gender (\%) & 19 & $918(56.9)$ & $5960(50.0)$ & $1.31[1.16,1.47]$ & 59.07 & 0.00 \\
Previous & 13 & $246(30.8)$ & $344(32.9)$ & $0.76[0.62,0.95]$ & 0.00 & 0.01 \\
$\quad$ abdominal & & & & & & \\
$\quad$ surgery (\%) & & & & & & \\
ASA $\geq 3(\%)$ & 16 & $286(30.2)$ & $1629(45.3)$ & $0.94[0.77,1.14]$ & 26.60 & 0.53 \\
T-stage $\geq 3(\%)$ & 18 & $831(54.9)$ & $6736(71.7)$ & $0.90[0.79,1.04]$ & 0.00 & 0.14 \\
\hline
\end{tabular}

N.O. number of, RCS robot-assisted colon surgery, LCS laparoscopic colon surgery, $O R$ odds ratio, $M D$ mean difference, $C I=$ confidence interval, $I^{2}$ heterogeneity

$p=0.04)$. We detected no differences regarding following outcomes: time to first flatus, intra-abdominal abscess rate, Clavien-Dindo complication rate I-III or IV-V, medical complications, postoperative bleeding, ileus, wound abscess, amount of lymph node harvest, estimated intraoperative blood loss or 30 days mortality rate (see Supplemental Digital Content Material 4, Figs. 1, 2, 3, 4, 5, 6, 7, 8, 9, 10, 11, 12, 13).

\section{Subgroup analysis: right-sided hemicolectomies}

Fourteen studies [12, 33, 35, 38, 39, 41-44, 46, 48-51] with a total number of 4198 patients $(\mathrm{RCS}=880(20.97 \%)$, $\mathrm{LCS}=3318(79.01 \%)$ underwent right-sided hemicolectomy. Conversion rates were lower in the RCS compared to the LCS group $\left(\mathrm{OR}=0.18,95 \% \mathrm{CI}=[0.10,0.30], I^{2}=12.72 \%\right.$, $p=0.00)$. The rate of anastomotic leakage was reduced in the RCS group (OR $=0.41,95 \%$ CI $\left.[0.19,0.88], I^{2}=0 \%, p=0.02\right)$. The amount of overall complication rates did not differ significantly between RCS and LCS group (OR $=0.85,95 \%$ CI [0.69, $1.04], I^{2}=25.26 \%, p=0.11$ ). Mean difference in operative time favorized LCS with considerable and significant heterogeneity $\left(\mathrm{MD}=57.45,95 \% \mathrm{CI}[38.47,76.42], I^{2}=94.46 \%, p=0.00\right)$. A significant reduction of length of stay with substantial heterogeneity was demonstrated in the RCS group (MD $=-0.91$,
95\% CI $\left.[-1.60,-0.22], I^{2}=73.74 \%, p=0.01\right)$. No difference between the surgical methods was found regarding to following outcomes: medical complications, Clavien-Dindo Complication rate I-V, 30 days mortality rate, abdominal abscess, wound abscess, postoperative bleeding, postoperative ileus, intraoperative blood loss, amount of harvested lymph nodes, time to regular diet or time to first flatus, presented in Supplemental Digital Content Material 5, Table 1.

\section{Sensitivity analysis}

We performed a supplementary sensitivity analysis on outcomes that initially presented with substantial or considerable heterogeneity $\left(I^{2}>50 \%\right)$ from main analyses. A significant advantage was found for RCS compared to LCS regarding length of stay $(\mathrm{MD}=-0.45,95 \% \mathrm{CI}$ $\left.[-0.73,-0.17], I^{2}=0 \%, p=0.00\right)$, amount of harvested lymph nodes (MD $=-0.96,95 \%$ CI $[-1.79,-0.14], I^{2}=0 \%$, $p=0.02)$ and intraoperative blood loss (MD $=-17.14,95 \%$ CI $\left.[-24.41,-9.87], I^{2}=19.10 \%, p=0.00\right)$. Operative time was in favor of LCS (MD $=41.99,95 \%$ CI [35.01, 48.96], $I^{2}=0 \%, p=0.00$ ). Time to regular diet and time to first flatus did not differ between the two surgical methods. See Supplemental Digital Content Material 6, Table 1. 


\begin{tabular}{|c|c|c|c|c|c|c|c|c|}
\hline \multirow[b]{2}{*}{ Study } & \multicolumn{4}{|c|}{ Robotic-assistedLaparoscopic } & \multirow[b]{2}{*}{$\begin{array}{c}\text { Favors } \\
\text { Robot }\end{array}$} & \multirow[b]{2}{*}{$\begin{array}{l}\text { Favors } \\
\text { Lap }\end{array}$} & \multirow{2}{*}{$\begin{array}{l}\text { Odds ratio } \\
\text { with } 95 \% \mathrm{Cl}\end{array}$} & \multirow{2}{*}{$\begin{array}{c}\text { Weight } \\
(\%)\end{array}$} \\
\hline & Yes & No & Yes & No & & & & \\
\hline Bertani $2011 \mathrm{M}$ & 1 & 33 & 1 & 29 & & & $0.88[0.05,14.69]$ & 2.75 \\
\hline Lim $2012 \mathrm{~L}$ & 0 & 34 & 2 & 144 & & & $0.84[0.04,17.85]$ & 2.53 \\
\hline Park 2012 R & 1 & 34 & 0 & 35 & & & $-3.09[0.12,78.41]$ & 1.28 \\
\hline Helvind 2013 M & 5 & 96 & 9 & 153 & & & $0.89[0.29, \quad 2.72]$ & 17.52 \\
\hline de'Angelis $2015 \mathrm{R}$ & 1 & 21 & 1 & 21 & & & $1.00[0.06,17.07]$ & 2.54 \\
\hline Cardinali 2016 R & 0 & 30 & 1 & 59 & & & $0.65[0.03,16.44]$ & 2.65 \\
\hline Jung $2016 \mathrm{M}$ & 1 & 50 & 1 & 160 & & & $3.20[0.20,52.10]$ & 1.26 \\
\hline Scotton 2018 R & 1 & 205 & 8 & 152 & & & $0.09\left[\begin{array}{ll}0.01, & 0.75\end{array}\right]$ & 23.89 \\
\hline Ngu 2018 R & 0 & 16 & 0 & 16 & & & $1.00[0.02,53.46]$ & 1.29 \\
\hline Mégevand 2018 R & 2 & 48 & 5 & 45 & & & $0.38[0.07,2.03]$ & 12.80 \\
\hline Spinoglio $2018 \mathrm{R}$ & 1 & 99 & 1 & 99 & & & $1.00[0.06,16.21]$ & 2.64 \\
\hline Polat $2019 \mathrm{M}$ & 2 & 122 & 6 & 127 & & & $0.35[0.07,1.75]$ & 15.19 \\
\hline Yozgatli $2019 \mathrm{R}$ & 0 & 35 & 3 & 58 & & & $0.24[0.01,4.69]$ & 6.76 \\
\hline Merola 2019 R & 1 & 93 & 1 & 93 & & & $1.00[0.06,16.23]$ & 2.64 \\
\hline Ozben $2020 \mathrm{R}$ & 0 & 38 & 2 & 78 & & & $0.41[0.02,8.70]$ & 4.28 \\
\hline Overall & & & & & & & $0.54[0.32,0.94]$ & \\
\hline \multicolumn{9}{|c|}{ Heterogeneity: $\mathrm{I}^{2}=0.00 \%, \mathrm{H}^{2}=1.00$} \\
\hline \multicolumn{9}{|c|}{ Test of $\theta_{i}=\theta_{j}: Q(14)=7.78, p=0.90$} \\
\hline \multicolumn{9}{|c|}{ Test of $\theta=0: z=-2.18, p=0.03$} \\
\hline & & & & & $1 / 4$ & 4 & 34 & \\
\hline
\end{tabular}

Fixed-effects Mantel-Haenszel model

$\mathrm{R}=$ right-sided colectomy, $\mathrm{L}=$ left sided colectomy, $\mathrm{M}=$ mixed resections, $\mathrm{I}^{2}=\mathrm{I}$-square, $\mathrm{Cl}=$ confidence interval

Fig. 2 Forest plot of anastomotic leakage

\section{Sensitivity analysis of studies with low risk of bias}

We included studies with low risk of bias and performed a sensitivity analysis across all outcomes. Conversion rates were in favor of RCS compared to LCS (OR $=0.31,95 \%$ CI [0.22, 0.43], $\left.I^{2}=50.17 \%, p=0.00\right)$. Except for shortened operative time in favor of LCS (MD $=71.00,95 \%$ CI [16.81, $125.19], I^{2}=98.56 \%, p=0.01$ ), no differences were observed across other outcomes between the RCS and LCS. See Supplemental Digital Content Material 7, Table 1.

\section{Meta-regression analysis}

Meta-regression analysis was performed in order to clarify a potential moderator effect on primary outcomes. We found following variables had a modifying effect on conversion rates in favor of robot-assisted surgery: increasing age $(\beta=-0.407$, $95 \%$ CI $[-0.727,-0.087], p=0.013)$, and a more recent year of publication $(\beta=-0.225,95 \%$ CI $[-0.452,0.002]$, $p=0.052$. Male gender $(\beta=0.431,95 \%$ CI $[0.045,0.818]$, $p=0.029)$ was an independent factor and favored conversion rates in the LCS group. The intracorporeal anastomosis technique was associated with a faster operative time in the RCS compared to the LCS group ( $\beta=-0.860,95 \%$ CI $[-1.576,-0.144], p=0.019)$, while a more recent year of publication favored operative times in the LCS group $(\beta=0.154,95 \%$ CI $[-0.003,0.312], p=0.054)$. The remaining co-variates did not have a moderating effect on other primary outcomes (anastomotic leakage or length of stay), see Supplemental Digital Content Material 8, Table 1.

\section{GRADE (quality of evidence) and publication bias assessment}

The quality of evidence was applied by GRADE assessment in the most important clinical outcomes (anastomotic leak, overall complication rate, conversion rate, intraoperative blood loss, length of hospital stay, Clavien-Dindo complication grade IV-V and 30 days mortality rate). We found the 


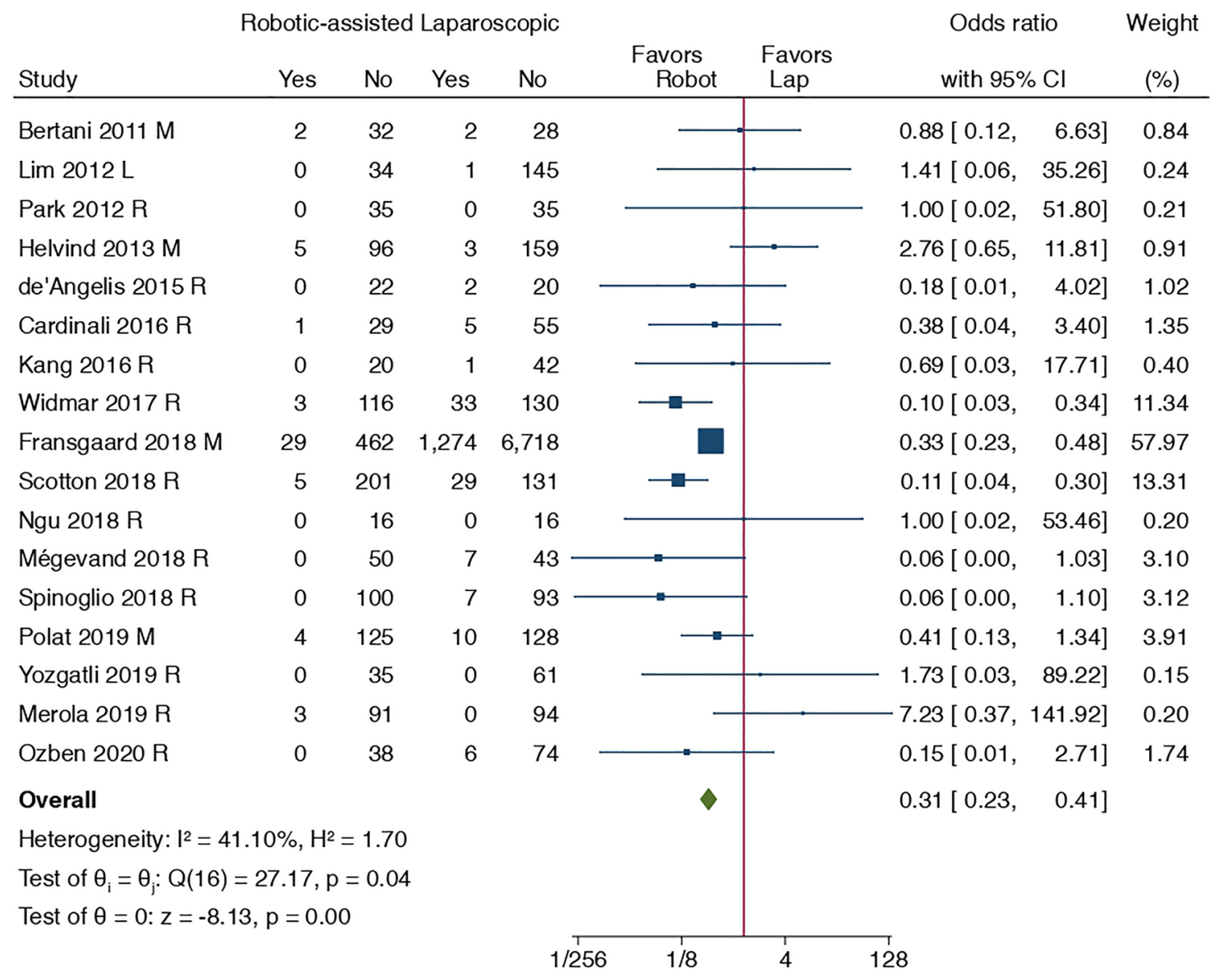

Fixed-effects Mantel-Haenszel model

$\mathrm{R}=$ right-sided colectomy, $\mathrm{L}=$ left sided colectomy, $\mathrm{M}=\operatorname{mixed}$ resections, $\mathrm{I}^{2}=\mathrm{I}$-square, $\mathrm{CI}=$

confidence interval

Fig. 3 Forest plot of conversion rate to open surgery

quality of evidence very low across all outcomes of interest mainly due to a serious risk of bias and/or inconsistency, see Table 3. In addition to the amount of intraoperative blood loss and harvested lymph nodes the included studies showed no signs of publication bias assessed by Funnel plots and Egger's regression test presented in Supplemental Digital Content Material 9, Table 1 and Figs. 1, 2, 3, 4, 5, 6, 7, 8, 9, $10,11,12,13,14,15,16$ and 17.

\section{Discussion}

To our knowledge, this systematic review and meta-analysis is the first conducted study to analyze robot-assisted compared to laparoscopic surgery for elective colonic cancer resections. It included 14,093 patients and aimed to evaluate the short-term clinical outcomes by the two surgical methods. Our study indicated that RCS was superior to LCS in terms of anastomotic leakage rate, overall complication rate, conversion to open surgery and time to regular diet. Despite a lack of prospective studies in the meta-analysis, our main results were affirmed by supplementary statistical analyses. These indicated robot-assisted method had several clinical benefits compared to laparoscopy in predominantly rightsided tumors, which may defend its standardized implementation for the future treatment of right-sided colonic cancer.

The development of minimally invasive surgery caused an increased transition from LCS to RCS in the last decade. However, our review found only one RCT on this topic, and the non-randomized studies included were of very low quality of evidence. Therefore, no high-quality scientific evidence for the routine use of RCS exists. Nevertheless, our results consistently favorized RCS in relation to several primary and secondary outcomes.

A reduction in anastomotic leakage rate may have multiple causes. Surgery performed in our study included two 


\begin{tabular}{|c|c|c|c|c|c|c|c|c|c|c|}
\hline \multirow[b]{2}{*}{ Study } & \multicolumn{3}{|c|}{ Robotic-assisted } & \multicolumn{3}{|c|}{ Laparoscopic } & \multirow[b]{2}{*}{$\begin{array}{l}\text { Favors } \\
\text { Robot }\end{array}$} & \multirow[b]{2}{*}{$\begin{array}{l}\text { Favors } \\
\text { Lap }\end{array}$} & \multirow{2}{*}{$\begin{array}{l}\text { Mean diff. } \\
\text { with } 95 \% \mathrm{Cl}\end{array}$} & \multirow{2}{*}{$\begin{array}{c}\text { Weight } \\
(\%)\end{array}$} \\
\hline & $\mathrm{N}$ & Mean & SD & $\mathrm{N}$ & Mean & SD & & & & \\
\hline Bertani $2011 \mathrm{M}$ & 34 & 5 & 3.25 & 30 & 5 & 2.25 & & & $0.00[-1.39,1.39]$ & 5.91 \\
\hline $\operatorname{Lim} 2012 \mathrm{~L}$ & 34 & 5.5 & 1.6 & 146 & 6.2 & 1.3 & & & $-0.70[-1.21,-0.19]$ & 7.02 \\
\hline Park 2012 R & 35 & 7.9 & 4.1 & 35 & 8.3 & 4.2 & & & $-0.40[-2.34,1.54]$ & 5.03 \\
\hline Helvind $2013 \mathrm{M}$ & 101 & 6.42 & 4.1666667 & 162 & 6.74 & 10.5 & & & $-0.32\left[\begin{array}{ll}-2.47, & 1.83]\end{array}\right.$ & 4.71 \\
\hline de'Angelis $2015 \mathrm{R}$ & 22 & 7.09 & 1.68 & 22 & 8.09 & 2.15 & & & $-1.00[-2.14,0.14]$ & 6.28 \\
\hline Cardinali $2016 \mathrm{R}$ & 30 & 6.83 & 2.36 & 60 & 8.02 & 4.88 & & & $-1.19[-3.04,0.66]$ & 5.18 \\
\hline Jung $2016 \mathrm{M}$ & 51 & 10 & 5.7 & 161 & 7.7 & 4.1 & & & $2.30[0.87,3.73]$ & 5.85 \\
\hline Kang 2016 R & 20 & 8.5 & 5.25 & 43 & 9 & 4.75 & & & $-0.50\left[\begin{array}{ll}-3.11, & 2.11]\end{array}\right.$ & 4.05 \\
\hline Haskins 2018 R & 89 & 4.4 & 2.4 & 2,405 & 5.2 & 4.7 & & & $-0.80\left[\begin{array}{ll}-1.78, & 0.18\end{array}\right]$ & 6.50 \\
\hline Scotton $2018 \mathrm{R}$ & 206 & 7.9 & 3.5 & 160 & 9.9 & 7.1 & & & $-2.00[-3.11,-0.89]$ & 6.32 \\
\hline Ngu $2018 \mathrm{R}$ & 16 & 4.5 & 2.75 & 16 & 4.5 & 3.25 & & & $0.00[-2.09,2.09]$ & 4.81 \\
\hline Mégevand $2018 \mathrm{R}$ & 50 & 5 & .5 & 50 & 8 & 1 & & & $-3.00[-3.31,-2.69]$ & 7.15 \\
\hline Spinoglio $2018 \mathrm{R}$ & 100 & 7.9 & 5.2 & 100 & 7.9 & 3.5 & & & $0.00[-1.23,1.23]$ & 6.15 \\
\hline Yozgatli $2019 \mathrm{R}$ & 35 & 6 & 3 & 61 & 6 & 3 & & & $0.00[-1.25,1.25]$ & 6.13 \\
\hline Merola 2019 R & 94 & 4 & 2 & 94 & 4 & 2 & & & $0.00[-0.57,0.57]$ & 6.96 \\
\hline Ceccarelli 2020 R & 26 & 7.2 & 1.5 & 29 & 8 & 2.9 & & & $-0.80[-2.04,0.44]$ & 6.13 \\
\hline Ozben $2020 \mathrm{R}$ & 38 & 7.2 & 3.1 & 80 & 7.9 & 4 & & & $-0.70[-2.14,0.74]$ & 5.82 \\
\hline \multicolumn{7}{|c|}{ Overall } & & & $-0.58[-1.37,0.21]$ & \\
\hline \multicolumn{11}{|c|}{ Heterogeneity: $\tau^{2}=2.25, I^{2}=91.10 \%, H^{2}=11.24$} \\
\hline \multicolumn{11}{|c|}{ Test of $\theta_{1}=\theta_{j}: Q(16)=179.83, p=0.00$} \\
\hline \multicolumn{11}{|c|}{ Test of $\theta=0: z=-1.43, p=0.15$} \\
\hline & & & & & & & -2 & 2 & 4 & \\
\hline
\end{tabular}

Random-effects DerSimonian-Laird model

$\mathrm{R}=$ right-sided colectomy, $\mathrm{L}=$ left sided colectomy, $\mathrm{M}=$ mixed resections, $\mathrm{I}^{2}=\mathrm{I}$-square, $\mathrm{CI}=$ confidence interval

Fig. 4 Forest plot of length of hospital stay

different anastomosis techniques (extra- or intracorporeal). In a supplementary meta-regression analysis the anastomotic technique did not have a modifying effect on the anastomotic leakage rate. Previous systematic literature and meta-analyses compared the anastomotic leakage rate in intra- and extracorporeal anastomoses techniques in minimally invasive colon surgery with divergent conclusions [16, 52-54]. Possible causes of an increased leakage rate in extracorporeal anastomosis techniques are caused by a greater mobilization and traction on the bowel mesentery [53]. This manipulation may induce a decreased blood supply to the anastomosis, with an increased risk of leakage. There is also a risk of injury to bowel serosa, and increased risk of perforation in the extracorporeal anastomosis technique $[16,53]$. A recent study by Emile et al. compared intra- and extracorporeal anastomoses techniques for benign and malignant conditions in minimally invasive right-sided colectomies. The rate of anastomotic leakage favorized the intracorporeal technique [16].

The risk of conversion in RCS versus LCS for colonic cancer surgery has been debated in previous meta-analyses with a general trend of lower conversion rates in RCS for right-sided resections $[14,15,55,56]$. No significant impact on conversion rates in the RCS group was demonstrated in the meta-regression analysis despite a statistical overrepresentation of patients who previously had undergone abdominal surgery. Besides the ROLARR study [57], the risk of conversion to open surgery from RCS or LCS has not been investigated in a prospective, randomized design. The ROLARR study was conducted on rectal cancer resections and found no differences in conversion rates between groups. However, the study reported a significant difference in male patients with higher BMI in favor of RCS. In a recent meta-analysis by Wee et al. there was no divergence in the risk of conversion in obese patients between the two surgical methods [58]. No preoperative patient selection in either of the surgical methods occurred in our study regarding BMI.

Operative times in RCS were longer compared to LCS in our study. Prolonged operative times in RCS can be attributed to several factors such as docking time, learning curve and more technically demanding procedures like intracorporeal suturing. It has previously been reported that a 


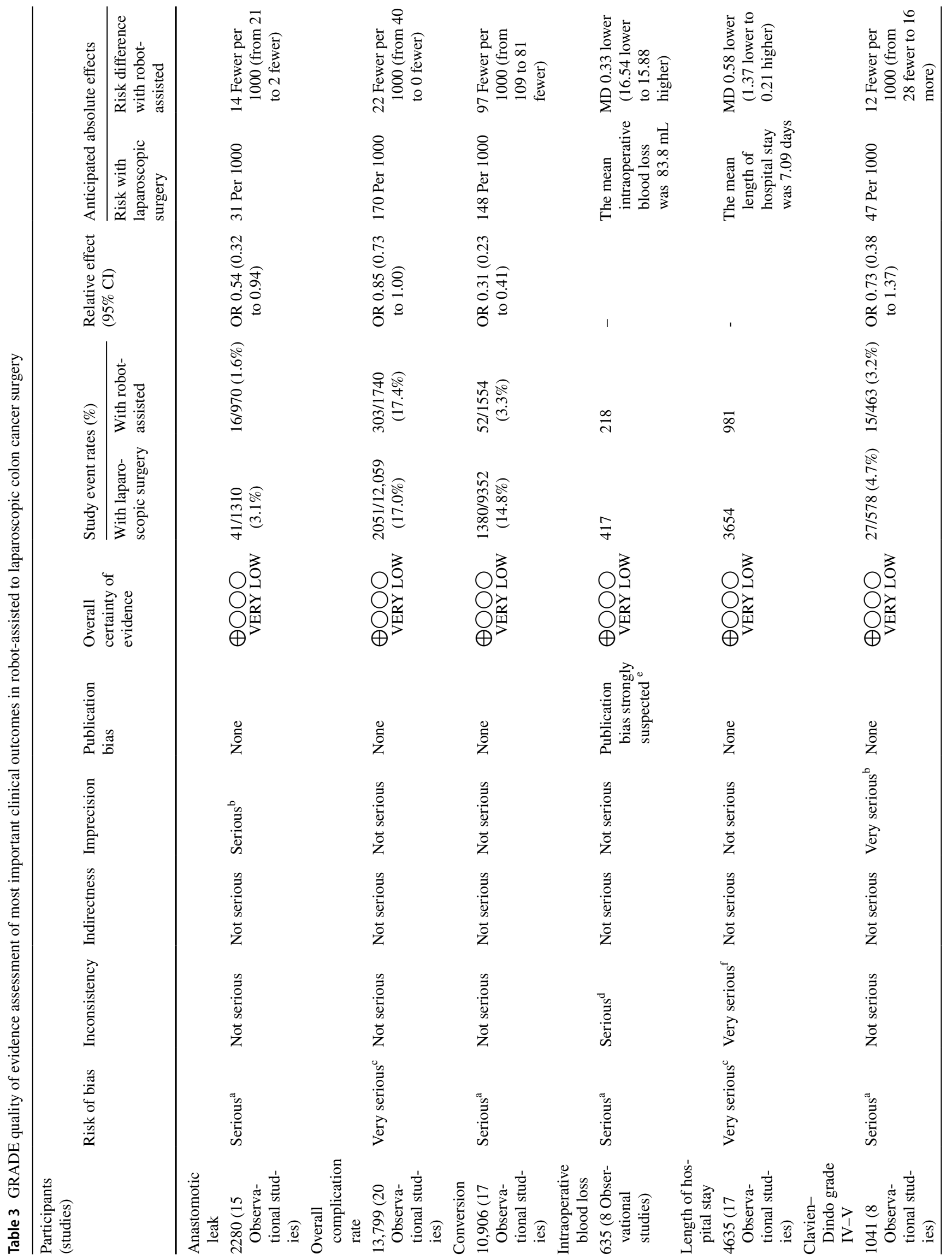


surgeon's learning curve influences surgery time. After 21 cases, the operative times in RCS and LCS in right-sided hemicolectomy for cancer were equalized [37]. We found the operation time was still prolonged in the RCS group after the implementation of CME surgery.

Overall complication rates and surgical morbidity has been described previously with varying results following RCS $[14,15,59,60]$. Several factors are influenced by this outcome, why it is difficult to provide a conclusive explanation. Study's data are heterogeneous and includes benign and malignant colorectal surgery, the results may be difficult to distinguish from each other and even misleading. The technical development has led to improved surgical procedures and reduced the risk of complications in robot-assisted surgery. It has several advantages over laparoscopic surgery and include a stable camera platform, the operator's possibility to control the camera independently, and the endowrist possibility of instruments [61]. The ever-increasing flexibility of the instruments makes it possible to perform complex procedures which either are impossible to perform or time-consuming by conventional laparoscopy. The tactile sense in open colorectal surgery is difficult to reproduce in minimally invasive surgery. However, RCS provides haptic feedback giving the surgeon a more "real" experience of the surgical procedure while performing intracorporeal suturing, manipulating the tissue and during dissection work [62]. This feature may help minimize the risk of intraoperative complications and reduce overall morbidity.

Time to first oral diet was reduced in the RCS group. This observation's significance can be debated since the reduction was minimal (mean difference $<1$ day) in relation to the high heterogeneity across studies. Previous studies have demonstrated a difference in length of stay, time to first flatus and oral intake in favor of RCS [13, 14, 55]. Despite divergent results, it may be assumed that RCS causes less tissue trauma due to better hemostasis technique and haptic feedback resulting in less manipulation of the organs.

The strengths of this study were the extensive literature search despite the inclusion of only one RCT. By supplementing the main results with subgroup-, sensitivity-, and meta-regression analyses we explored potential sources of outcomes with substantial heterogeneity. In a subgroup analysis of right-sided colectomies, we demonstrated no difference in the overall complication rate and time to regular diet between the two groups compared to the results from primary analysis, while length of stay was reduced by approximately one day in the RCS group. Due to a limited number of studies performing left-sided colectomies conducting a subgroup analysis was impossible. We conducted a sensitivity analysis with the exclusion of outlier studies with extreme effect sizes, as a consequence of substantial and considerable heterogeneity that originated in some of our primary and secondary outcomes. An additional significant 
difference was demonstrated regarding the intraoperative blood loss and amount of harvested lymph nodes in favor of RCS.

A supplemental meta-regression analysis was performed to verify any moderator effect on primary outcomes. Higher age was proved to reduce the risk of conversion in robot-assisted surgery, while the male sex had a greater advantage if operated by the laparoscopic method. Additionally, an intracorporeal anastomotic technique predicted a reduction in operative time in the RCS group probably as a direct consequence of a more accessible approach to intracorporeal suturing than the LCS group. A more recent year of publication impacted conversion rates and was reduced in the RCS group, while the operative time constantly favored laparoscopic surgery across all years included in this meta-analysis. By a summary analysis of literature available, we identified several clinically relevant factors favoring RCS compared to LCS. This new information can be utilized for a transformation process and replace laparoscopic surgery in the treatment of colon cancer. Although some of the outcomes had little clinical significance (e.g., reduction in length of stay and time to initiation of regular diet), this is believed to be indirectly correlated to the lower overall complication-, conversion-, and anastomotic leakage rate favoring RCS.

The substantial limitation of this study is the inclusion of studies with a non-randomized design resulting in a risk of selection bias. A patient selection in favor of RCS may occur during the implementation phase explained by the differences in some of the preoperative demographic and clinical outcomes between RCS and LCS. The studies included in our meta-analysis were of a different design regarding the type of surgery and anastomotic techniques. A plausible explanation of high heterogeneity deriving in some of our outcomes of interest can be explained by this diversity. Since most of our studies are of an observational design the evidence level is very low, and risk of bias assessment of serious concern. Our results may likewise have been over- or underestimated regarding following outcomes: length of stay, time to first flatus, time to regular diet, rate of intraoperative bleeding thereby causing information bias.

In conclusion, the present meta-analysis indicates that RCS for colon cancer is superior to LCS in several intraand postoperative outcomes with regard to a very low level of evidence and serious risk of bias. Our result can thus only be applied to right-sided resections due to lack of studies comparing exclusively left-sided colon surgery, why this surgical method cannot be recommended by default for this group of patients. Due to the considerable divergence between anastomosis techniques in right-sided resections, there is a need for prospective randomized studies examining intra- and extracorporeal techniques in
RCS. It is believed that postoperative complication rates and time of convalescence are improved by intracorporeal anastomosis technique in RCS.

Supplementary Information The online version contains supplementary material available at https://doi.org/10.1007/s00464-021-08782-7.

Acknowledgements We would like to thank Caroline Moos for critical review and proofreading of this manuscript and research librarian Mette Brandt Eriksen, University of Southern Denmark for the assistance of performing the literature search strategy.

Funding The study was supported by Aage and Johanne Louis-Hansen Fund (20-2B-6796).Study register This study was prospectively registered in PROSPERO, identifier CRD42020211681.

Data availability Data extracted and included in the systematic review and meta-analysis can be handed out on request from the corresponding author.

Disclosures Pedja Cuk, Mie Dilling Kjær, Mark Bremholm Ellebæk, Michael Festersen Nielsen, Christian Backer Mogensen, Andreas Kristian Pedersen have no conflicts of interest or financial ties to disclose.

Open Access This article is licensed under a Creative Commons Attribution 4.0 International License, which permits use, sharing, adaptation, distribution and reproduction in any medium or format, as long as you give appropriate credit to the original author(s) and the source, provide a link to the Creative Commons licence, and indicate if changes were made. The images or other third party material in this article are included in the article's Creative Commons licence, unless indicated otherwise in a credit line to the material. If material is not included in the article's Creative Commons licence and your intended use is not permitted by statutory regulation or exceeds the permitted use, you will need to obtain permission directly from the copyright holder. To view a copy of this licence, visit http://creativecommons.org/licenses/by/4.0/.

\section{References}

1. Weber PA, Merola S, Wasielewski A, Ballantyne GH (2002) Telerobotic-assisted laparoscopic right and sigmoid colectomies for benign disease. Dis Colon Rectum 45(12):1689-1694, discussion 95-96. https://doi.org/10.1007/s10350-004-7261-2

2. Sun Y, Xu H, Li Z, Han J, Song W, Wang J et al (2016) Robotic versus laparoscopic low anterior resection for rectal cancer: a meta-analysis. World J Surg Oncol 14:61. https://doi.org/10.1186/ s12957-016-0816-6

3. Lee SH, Kim DH, Lim SW (2018) Robotic versus laparoscopic intersphincteric resection for low rectal cancer: a systematic review and meta-analysis. Int J Colorectal Dis 33(12):1741-1753. https://doi.org/10.1007/s00384-018-3145-0

4. Holmer C, Kreis ME (2018) Systematic review of robotic low anterior resection for rectal cancer. Surg Endosc 32(2):569-581. https://doi.org/10.1007/s00464-017-5978-y

5. Sivathondan PC, Jayne DG (2018) The role of robotics in colorectal surgery. Ann R Coll Surg Engl 100(Suppl 7):42-53. https:// doi.org/10.1308/rcsann.supp2.42 
6. Hasegawa H, Kabeshima Y, Watanabe M, Yamamoto S, Kitajima M (2003) Randomized controlled trial of laparoscopic versus open colectomy for advanced colorectal cancer. Surg Endosc 17(4):636-640. https://doi.org/10.1007/s00464-002-8516-4

7. Braga M, Vignali A, Zuliani W, Frasson M, Di Serio C, Di Carlo V (2005) Laparoscopic versus open colorectal surgery: costbenefit analysis in a single-center randomized trial. Ann Surg 242(6):890-895, discussion 5-6. https://doi.org/10.1097/01.sla. 0000189573.23744 .59

8. Huang YM, Lee YW, Huang YJ, Wei PL (2020) Comparison of clinical outcomes between laparoscopic and open surgery for leftsided colon cancer: a nationwide population-based study. Sci Rep 10(1):75. https://doi.org/10.1038/s41598-019-57059-6

9. Shibata J, Ishihara S, Tada N, Kawai K, Tsuno NH, Yamaguchi $\mathrm{H}$ et al (2015) Surgical stress response after colorectal resection: a comparison of robotic, laparoscopic, and open surgery. Tech Coloproctol 19(5):275-280. https://doi.org/10.1007/ s10151-014-1263-4

10. Hildebrandt U, Kessler K, Plusczyk T, Pistorius G, Vollmar B, Menger MD (2003) Comparison of surgical stress between laparoscopic and open colonic resections. Surg Endosc 17(2):242-246. https://doi.org/10.1007/s00464-001-9148-9

11. Veenhof AA, Vlug MS, van der Pas MH, Sietses C, van der Peet DL, de Lange-de Klerk ES et al (2012) Surgical stress response and postoperative immune function after laparoscopy or open surgery with fast track or standard perioperative care: a randomized trial. Ann Surg 255(2):216-221. https://doi.org/10.1097/SLA. 0b013e31824336e2

12. Park JS, Choi GS, Park SY, Kim HJ, Ryuk JP (2012) Randomized clinical trial of robot-assisted versus standard laparoscopic right colectomy. Br J Surg 99(9):1219-1226. https://doi.org/10.1002/ bjs. 8841

13. Ng KT, Tsia AKV, Chong VYL (2019) Robotic versus conventional laparoscopic surgery for colorectal cancer: a systematic review and meta-analysis with trial sequential analysis. World J Surg 43(4):1146-1161. https://doi.org/10.1007/ s00268-018-04896-7

14. Trastulli S, Cirocchi R, Desiderio J, Coratti A, Guarino S, Renzi $\mathrm{C}$ et al (2015) Robotic versus laparoscopic approach in colonic resections for cancer and benign diseases: systematic review and meta-analysis. PLoS ONE 10(7):e0134062. https://doi.org/10. 1371/journal.pone.0134062

15. Genova P, Pantuso G, Cipolla C, Latteri MA, Abdalla S, Paquet JC et al (2020) Laparoscopic versus robotic right colectomy with extra-corporeal or intra-corporeal anastomosis: a systematic review and meta-analysis. Langenbecks Arch Surg. https://doi. org/10.1007/s00423-020-01985-x

16. Emile SH, Elfeki H, Shalaby M, Sakr A, Bassuni M, Christensen $P$ et al (2019) Intracorporeal versus extracorporeal anastomosis in minimally invasive right colectomy: an updated systematic review and meta-analysis. Tech Coloproctol 23(11):1023-1035. https:// doi.org/10.1007/s10151-019-02079-7

17. Moher D, Liberati A, Tetzlaff J, Altman DG, PRISMA Group (2009) Preferred reporting items for systematic reviews and metaanalyses: the PRISMA statement. PLoS Med 6(7):e1000097. https://doi.org/10.1371/journal.pmed.1000097

18. Stroup DF, Berlin JA, Morton SC, Olkin I, Williamson GD, Rennie D et al (2000) Meta-analysis of observational studies in epidemiology: a proposal for reporting. Meta-analysis Of Observational Studies in Epidemiology (MOOSE) Group. JAMA 283(15):20082012. https://doi.org/10.1001/jama.283.15.2008

19. Dindo D, Demartines N, Clavien PA (2004) Classification of surgical complications: a new proposal with evaluation in a cohort of 6336 patients and results of a survey. Ann Surg 240(2):205-213. https://doi.org/10.1097/01.sla.0000133083.54934.ae
20. Sterne JAC, Savovic J, Page MJ, Elbers RG, Blencowe NS, Boutron I et al (2019) RoB2: a revised tool for assessing risk of bias in randomised trials. BMJ 366:14898. https://doi.org/10.1136/ bmj.14898

21. Sterne JA, Hernan MA, Reeves BC, Savovic J, Berkman ND, Viswanathan $\mathrm{M}$ et al (2016) ROBINS-I: a tool for assessing risk of bias in non-randomised studies of interventions. BMJ 355:i4919. https://doi.org/10.1136/bmj.i4919

22. McGuinness LA, Higgins JPT (2020) Risk-of-bias VISualization (Robvis): an R package and Shiny web app for visualizing riskof-bias assessments. Res Synth Methods. https://doi.org/10.1002/ jrsm. 1411

23. Guyatt G, Oxman AD, Akl EA, Kunz R, Vist G, Brozek J et al (2011) GRADE guidelines: 1. Introduction-GRADE evidence profiles and summary of findings tables. J Clin Epidemiol 64(4):383-394. https://doi.org/10.1016/j.jclinepi.2010.04.026

24. Hozo SP, Djulbegovic B, Hozo I (2005) Estimating the mean and variance from the median, range, and the size of a sample. BMC Med Res Methodol 5:13. https://doi.org/10.1186/1471-2288-5-13

25. Higgins JP, Thompson SG, Deeks JJ, Altman DG (2003) Measuring inconsistency in meta-analyses. BMJ 327(7414):557-560. https://doi.org/10.1136/bmj.327.7414.557

26. Cumpston M, Li T, Page MJ, Chandler J, Welch VA, Higgins JP et al (2019) Updated guidance for trusted systematic reviews: a new edition of the Cochrane Handbook for Systematic Reviews of Interventions. Cochrane Database Syst Rev 10:ED000142. https:// doi.org/10.1002/14651858.ED000142

27. Egger M, Davey Smith G, Schneider M, Minder C (1997) Bias in meta-analysis detected by a simple, graphical test. BMJ 315(7109):629-634. https://doi.org/10.1136/bmj.315.7109.629

28. Simmonds M (2015) Quantifying the risk of error when interpreting funnel plots. Syst Rev 4:24. https://doi.org/10.1186/ s13643-015-0004-8

29. Bertani E, Chiappa A, Biffi R, Bianchi PP, Radice D, Branchi $\mathrm{V}$ et al (2011) Assessing appropriateness for elective colorectal cancer surgery: clinical, oncological, and quality-of-life shortterm outcomes employing different treatment approaches. Int J Colorectal Dis 26(10):1317-1327. https://doi.org/10.1007/ s00384-011-1270-0

30. Lim DR, Min BS, Kim MS, Alasari S, Kim G, Hur H et al (2013) Robotic versus laparoscopic anterior resection of sigmoid colon cancer: comparative study of long-term oncologic outcomes. Surg Endosc 27(4):1379-1385. https://doi.org/10.1007/ s00464-012-2619-3

31. Helvind NM, Eriksen JR, Mogensen A, Tas B, Olsen J, Bundgaard $\mathrm{M}$ et al (2013) No differences in short-term morbidity and mortality after robot-assisted laparoscopic versus laparoscopic resection for colonic cancer: a case-control study of 263 patients. Surg Endosc 27(7):2575-2580. https://doi.org/10.1007/ s00464-013-2792-z

32. Morpurgo E, Contardo T, Molaro R, Zerbinati A, Orsini C, D'Annibale A (2013) Robotic-assisted intracorporeal anastomosis versus extracorporeal anastomosis in laparoscopic right hemicolectomy for cancer: a case control study. J Laparoendosc Adv Surg Tech A 23(5):414-417. https://doi.org/10.1089/lap.2012. 0404

33. de'Angelis N, Alghamdi S, Renda A, Azoulay D, Brunetti F (2015) Initial experience of robotic versus laparoscopic colectomy for transverse colon cancer: a matched case-control study. World J Surg Oncol 13:295. https://doi.org/10.1186/s12957-015-0708-1

34. Guerrieri M, Campagnacci R, Sperti P, Belfiori G, Gesuita R, Ghiselli R (2015) Totally robotic vs 3D laparoscopic colectomy: a single centers preliminary experience. World J Gastroenterol 21(46):13152-13159. https://doi.org/10.3748/wjg.v21.i46. 13152 
35. Kang J, Park YA, Baik SH, Sohn SK, Lee KY (2016) A comparison of open, laparoscopic, and robotic surgery in the treatment of right-sided colon cancer. Surg Laparosc Endosc Percutan Tech 26(6):497-502. https://doi.org/10.1097/SLE.0000000000000331

36. Jung YB, Kang J, Park EJ, Baik SH, Lee KY (2016) Time to initiation of adjuvant chemotherapy in colon cancer: comparison of open, laparoscopic, and robotic surgery. J Laparoendosc Adv Surg Tech A 26(10):799-805. https://doi.org/10.1089/lap.2016. 0293

37. de'Angelis N, Lizzi V, Azoulay D, Brunetti F (2016) Robotic versus laparoscopic right colectomy for colon cancer: analysis of the initial simultaneous learning curve of a surgical fellow. J Laparoendosc Adv Surg Tech A 26(11):882-892. https://doi.org/ 10.1089/lap.2016.0321

38. Cardinali L, Belfiori G, Ghiselli R, Ortenzi M, Guerrieri M (2016) Robotic versus laparoscopic right colectomy for cancer: shortterm outcomes and influence of Body Mass Index on conversion rate. Minerva Chir 71(4):217-222

39. Widmar M, Keskin M, Strombom P, Beltran P, Chow OS, Smith JJ et al (2017) Lymph node yield in right colectomy for cancer: a comparison of open, laparoscopic and robotic approaches. Colorectal Dis 19(10):888-894. https://doi.org/10.1111/codi.13786

40. Kim JC, Lee JL, Yoon YS, Kim CW, Park IJ, Lim SB (2018) Robotic left colectomy with complete mesocolectomy for splenic flexure and descending colon cancer, compared with a laparoscopic procedure. Int J Med Robot 14(5):e1918. https://doi.org/ 10.1002/rcs. 1918

41. Scotton G, Contardo T, Zerbinati A, Tosato SM, Orsini C, Morpurgo E (2018) From laparoscopic right colectomy with extracorporeal anastomosis to robot-assisted intracorporeal anastomosis to totally robotic right colectomy for cancer: the evolution of robotic multiquadrant abdominal surgery. J Laparoendosc Adv Surg Tech A 28(10):1216-1222. https://doi.org/ 10.1089/lap.2017.0693

42. Ngu JC, Ng YY (2018) Robotics confers an advantage in right hemicolectomy with intracorporeal anastomosis when matched against conventional laparoscopy. J Robot Surg 12(4):647-653. https://doi.org/10.1007/s11701-018-0793-6

43. Spinoglio G, Bianchi PP, Marano A, Priora F, Lenti LM, Ravazzoni F et al (2018) Robotic versus laparoscopic right colectomy with complete mesocolic excision for the treatment of colon cancer: perioperative outcomes and 5-year survival in a consecutive series of 202 patients. Ann Surg Oncol 25(12):3580-3586. https:// doi.org/10.1245/s10434-018-6752-7

44. Megevand JL, Amboldi M, Lillo E, Lenisa L, Ganio E, Ambrosi A et al (2019) Right colectomy: consecutive 100 patients treated with laparoscopic and robotic technique for malignancy. Cumulative experience in a single centre. Updates Surg 71(1):151-156. https://doi.org/10.1007/s13304-018-0599-0

45. Fransgaard T, Pinar I, Thygesen LC, Gogenur I (2018) Association between robot-assisted surgery and resection quality in patients with colorectal cancer. Surg Oncol 27(2):177-184. https://doi.org/ 10.1016/j.suronc.2018.03.003

46. Haskins IN, Ju T, Skancke M, Kuang X, Amdur RL, Brody F et al (2018) Right colon resection for colon cancer: does surgical approach matter? J Laparoendosc Adv Surg Tech A 28(10):12021206. https://doi.org/10.1089/lap.2018.0148

47. Polat F, Willems LH, Dogan K, Rosman C (2019) The oncological and surgical safety of robot-assisted surgery in colorectal cancer: outcomes of a longitudinal prospective cohort study. Surg Endosc 33(11):3644-3655. https://doi.org/10.1007/s00464-018-06653-2

48. Yozgatli TK, Aytac E, Ozben V, Bayram O, Gurbuz B, Baca B et al (2019) Robotic complete mesocolic excision versus conventional laparoscopic hemicolectomy for right-sided colon cancer. J Laparoendosc Adv Surg Tech A 29(5):671-676. https://doi.org/ 10.1089/lap.2018.0348
49. Ozben V, de Muijnck C, Sengun B, Zenger S, Agcaoglu O, Balik E et al (2020) Robotic complete mesocolic excision for transverse colon cancer can be performed with a morbidity profile similar to that of conventional laparoscopic colectomy. Tech Coloproctol 24(10):1035-1042. https://doi.org/10.1007/s10151-020-02249-y

50. Ceccarelli G, Costa G, Ferraro V, De Rosa M, Rondelli F, Bugiantella W (2020) Robotic or three-dimensional (3D) laparoscopy for right colectomy with complete mesocolic excision (CME) and intracorporeal anastomosis? A propensity score-matching study comparison. Surg Endosc. https://doi.org/10.1007/ s00464-020-07600-w

51. Merola G, Sciuto A, Pirozzi F, Andreuccetti J, Pignata G, Corcione $\mathrm{F}$ et al (2020) Is robotic right colectomy economically sustainable? A multicentre retrospective comparative study and cost analysis. Surg Endosc 34(9):4041-4047. https://doi.org/10.1007/ s00464-019-07193-z

52. Wu Q, Jin C, Hu T, Wei M, Wang Z (2017) Intracorporeal versus extracorporeal anastomosis in laparoscopic right colectomy: a systematic review and meta-analysis. J Laparoendosc Adv Surg Tech A 27(4):348-357. https://doi.org/10.1089/lap.2016.0485

53. Brown RF, Cleary RK (2020) Intracorporeal anastomosis versus extracorporeal anastomosis for minimally invasive colectomy. $\mathrm{J}$ Gastrointest Oncol 11(3):500-507. https://doi.org/10.21037/jgo. 2019.12.02

54. Aiolfi A, Bona D, Guerrazzi G, Bonitta G, Rausa E, Panizzo V et al (2020) Intracorporeal versus extracorporeal anastomosis in laparoscopic right colectomy: an updated systematic review and cumulative meta-analysis. J Laparoendosc Adv Surg Tech A 30(4):402-412. https://doi.org/10.1089/lap.2019.0693

55. Solaini L, Bazzocchi F, Cavaliere D, Avanzolini A, Cucchetti A, Ercolani G (2018) Robotic versus laparoscopic right colectomy: an updated systematic review and meta-analysis. Surg Endosc 32(3):1104-1110. https://doi.org/10.1007/s00464-017-5980-4

56. Chang YS, Wang JX, Chang DW (2015) A meta-analysis of robotic versus laparoscopic colectomy. J Surg Res 195(2):465474. https://doi.org/10.1016/j.jss.2015.01.026

57. Jayne D, Pigazzi A, Marshall H, Croft J, Corrigan N, Copeland J et al (2017) Effect of robotic-assisted vs conventional laparoscopic surgery on risk of conversion to open laparotomy among patients undergoing resection for rectal cancer: the ROLARR randomized clinical trial. JAMA 318(16):1569-1580. https://doi.org/10.1001/ jama.2017.7219

58. Wee IJY, Kuo LJ, Ngu JC (2019) The impact of robotic colorectal surgery in obese patients: a systematic review, meta-analysis, and meta-regression. Surg Endosc 33(11):3558-3566. https://doi.org/ 10.1007/s00464-019-07000-9

59. Waters PS, Cheung FP, Peacock O, Heriot AG, Warrier SK, O'Riordain DS et al (2020) Successful patient-oriented surgical outcomes in robotic vs laparoscopic right hemicolectomy for cancer-a systematic review. Colorectal Dis 22(5):488-499. https:// doi.org/10.1111/codi.14822

60. Rausa E, Kelly ME, Asti E, Aiolfi A, Bonitta G, Bonavina L (2019) Right hemicolectomy: a network meta-analysis comparing open, laparoscopic-assisted, total laparoscopic, and robotic approach. Surg Endosc 33(4):1020-1032. https://doi.org/10.1007/ s00464-018-6592-3

61. Karcz WK, von Braun W (2016) Minimally invasive surgery for the treatment of colorectal cancer. Visc Med 32(3):192-198. https://doi.org/10.1159/000445815

62. Roy S, Evans C (2016) Overview of robotic colorectal surgery: current and future practical developments. World J Gastrointest Surg 8(2):143-150. https://doi.org/10.4240/wjgs.v8.i2.143

Publisher's Note Springer Nature remains neutral with regard to jurisdictional claims in published maps and institutional affiliations. 